\title{
Generalized parton distributions of the pion in light-front dynamics
}

\section{Bernard BAKKER ${ }^{*}$}

Department of Physics and Astronomy, Vrije Universiteit, Amsterdam

E-mail: blg.bakkerefew.vu.nl

\section{Chueng-Ryong Jl}

North Carolina State University

E-mail: crjiencsu.edu

Deeply virtual Compton scattering has been recognized as a tool to obtain information about hadronic wave functions from experimental data. Light-front dynamics provides boost-invariant and process-independent wave functions, which makes it the preferred theoretical tool to analyze those data. The purpose of the present work is to apply it to a model of the pion and check its usefulness in a situation where all ingredients of the calculations are fully under control.

LIGHT CONE 2008 Relativistic Nuclear and Particle Physics

July 7-11 2008

Mulhouse, France

\footnotetext{
* Speaker.
} 


\section{Motivation}

Compton scattering is a powerful tool to study hadronic structure, because it probes hadrons through a coupling to two electromagnetic currents, providing additional information compared to e.g. form-factor data. Deeply virtual Compton scattering (DVCS) [1,2] in particular furnishes information on the structure of bound states, as the amplitudes at large momentum transfers can be written as a convolution of a hard-scattering amplitude calculable in perturbative QCD, and a process-independent 'soft' distribution function [3]:

$$
\mathscr{A}=\int_{-1}^{1} \mathrm{~d} x T\left(x, \zeta, Q^{2}\right) \mathscr{H}(x, \zeta, t),
$$

where $T$ is the hard scattering amplitude and $\mathscr{H}$ is the soft part. Those functions corresponding to $\mathscr{H}$ are called generalized parton distributions (GPDs) in the case of DVCS. For a review see for instance [4].

Light-front dynamics (LFD) is particularly suited to deal with such a situation, as it allows for a factorization of the wave function of a system into a factor for its overall motion and a wave function that describes its internal structure. Moreover, LFD makes use of the Fock-space expansion, so the role of higher-Fock sectors in a particular process can be studied.

However, a word of caution is appropriate, because in LFD some subtleties may prevent its straightforward application. An example of such treacherous points [5] occurs in the GPD of the pion too, namely an arc contribution [6].

For values of the momentum transfer going to infinity, the 'handbag' diagram is proven to be dominant. In this work, we discuss this dominance for non-asymptotic values of the momentum transfer in an analytically solvable scalar model for the pion GPD and remark on the reason why an arc contribution does occur in a similar model where the quarks are treated as spin-1/2 constituents.

\section{Model calculations}

In order to avoid any unnecessary complications we study the GPD of the 'pion', decribed as a scalar particle with scalar constituents [7] in 1+1 dimensions. (The limitation to $1+1$ dimensions can be lifted without complicating the calculations if a kinematics is chosen in which the external particles have vanishing perpendicular momenta.)

\subsection{Kinematics}

Using the convention $p^{ \pm}=\left(p^{0} \pm p^{3}\right) / \sqrt{ } 2$ we write the momenta of the absorbed virtual photon $(q)$, emitted real photon $\left(q^{\prime}\right)$, incoming pion $(p)$ and outgoing pion $\left(p^{\prime}\right)$ as follows:

$$
\begin{aligned}
& q=\left(-\zeta p^{+}, \frac{Q^{2}}{2 \zeta p^{+}}\right), \quad q^{\prime}=\left(0, \frac{1}{2 p^{+}}\left(-\frac{\zeta}{1-\zeta} M^{2}+\frac{Q^{2}}{\zeta}\right)\right), \\
& p=\left(p^{+}, \frac{M^{2}}{2 p^{+}}\right), \quad p^{\prime}=\left((1-\zeta) p^{+}, \frac{M^{2}}{2(1-\zeta) p^{+}}\right),
\end{aligned}
$$

where $M$ is the mass of the pion and $\zeta$ is the skewness parameter. For the Mandelstam variables we then find

$$
s=(1-\zeta) M^{2}+\frac{1-\zeta}{\zeta} Q^{2}, \quad t=-\frac{\zeta^{2}}{1-\zeta} M^{2}, \quad u=\frac{M^{2}}{1-\zeta}-\frac{Q^{2}}{\zeta} .
$$



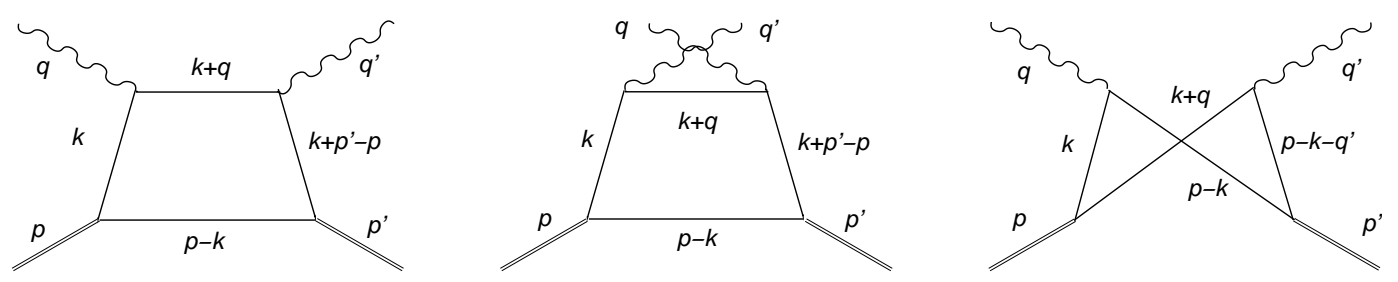

Figure 1: Handbag, crossed handbag, and cats ears diagrams

In this kinematics, we calculate the three diagrams shown in Fig. 1 that contribute to the DVCS amplitude, below the breakup threshold $s=4 m^{2}$ which limits $Q^{2}$ to $0<Q^{2}<Q_{\max }^{2}$ with $Q_{\max }^{2}=4 \zeta m^{2} /(1-\zeta)-(1-\zeta) M^{2}$. In principle, the 'seagull' diagram is needed for the scalar constituents interacting with the gauge bosons to fulfill the gauge invariance of the Compton amplitude. However, the 'seagull' diagram does not contribute to the GPD as shown in Ref. [9], therefore we left it out.

\subsection{Covariant calculation}

In the covariant calculation, we followed the usual path of Feynman parametrization and Wick rotation to analytically perform the integrals over the four momentum. If we write for the amplitude

$$
\mathscr{A}=\int \frac{\mathrm{d}^{2} k}{(2 \pi)^{2}} \frac{N}{D}, \quad D=\left(k_{1}^{2}-m^{2}+i \varepsilon\right)\left(k_{2}^{2}-m^{2}+i \varepsilon\right)\left(k_{3}^{2}-m^{2}+i \varepsilon\right)\left(k_{4}^{2}-m^{2}+i \varepsilon\right),
$$

then we end up with an integral over the Feyman parameters with the denominator $\left(M_{\mathrm{cov}}^{2}\right)^{3}$. For the three diagrams we considered, we have

$$
\begin{aligned}
& M_{s t}^{2}=m^{2}-\alpha_{1}\left(\alpha_{2}+\alpha_{4}\right) M^{2}+\alpha_{2} \alpha_{3} Q^{2}-\alpha_{1} \alpha_{3} s-\alpha_{2} \alpha_{4} t \\
& M_{u t}^{2}=m^{2}-\alpha_{1}\left(\alpha_{2}+\alpha_{4}\right) M^{2}+\alpha_{2} \alpha_{3} Q^{2}-\alpha_{1} \alpha_{3} u-\alpha_{2} \alpha_{4} t, \\
& M_{s u}^{2}=m^{2}-\left(\alpha_{1} \alpha_{2}+\alpha_{3} \alpha_{4}\right) M^{2}+\alpha_{2} \alpha_{3} Q^{2}-\alpha_{1} \alpha_{3} s-\alpha_{2} \alpha_{4} u,
\end{aligned}
$$

where $m$ is the constituent mass. In the model with scalar constituents, $N=1$.

\subsection{LF time-ordered diagrams}

The LF time-ordered diagrams are obtained by integrating over $k^{-}$first and picking up the residues of the poles in the denominator $D=\prod_{i}\left(k_{i}^{2}-m_{i}^{2}+i \varepsilon\right)=\prod_{i} 2 k_{i}^{+}\left(k_{i}^{-}-H_{i}\right)$.
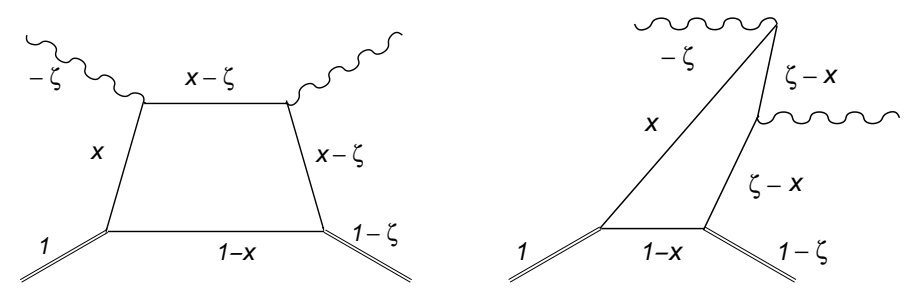

Figure 2: Valence (DGLAP) and nonvalence (ERBL) contributions to the handbag diagram. 

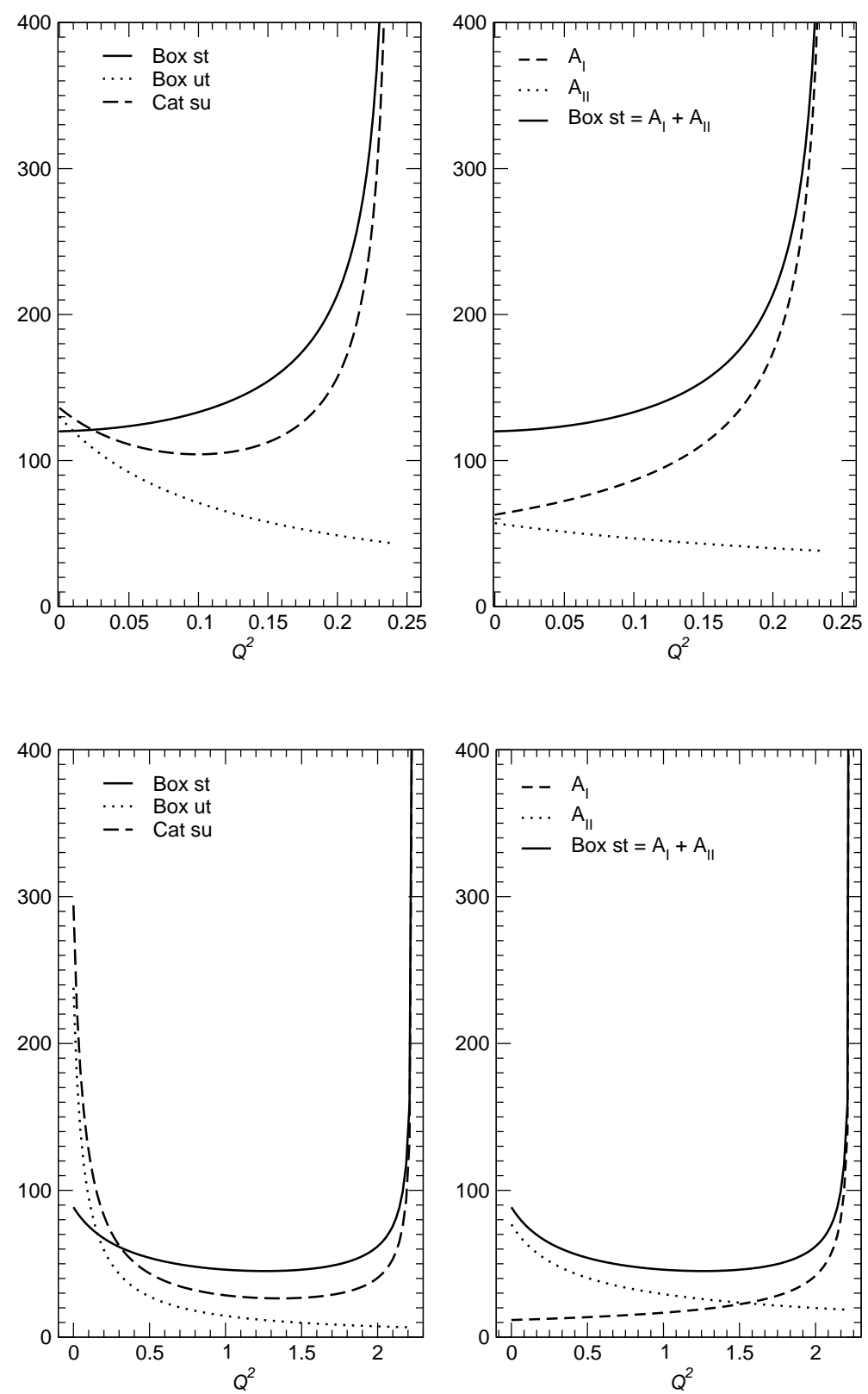

Figure 3: Covariant amplitudes (left) and LF contributions to the Box st (right), for $\zeta=0.5$ above and $\zeta=0.9$ below.

For our kinematics $p^{+}<p^{+}$, we find two domains that correspond to the well-known DGLAP and ERBL regions: DGLAP, $\zeta p^{+} \leq k^{+}<p^{+}$, ERBL, $0 \leq k^{+}<\zeta p^{+}$. For the covariant handbag diagram, we show the corresponding LF diagrams in Fig. 2.

For the values $\zeta=0.5$ and 0.9 , we show the amplitudes in Fig. 3. We took $M=0.14$ for the pion mass and $m=0.25$ for the constituent mass. The results of the LF calculations for the three diagrams considered coincide with the covariant results. We show that explicitly in the r.h. panels of Fig. 3. The 1.h. panels show that the handbag diagram denoted by 'Box st' is in this 


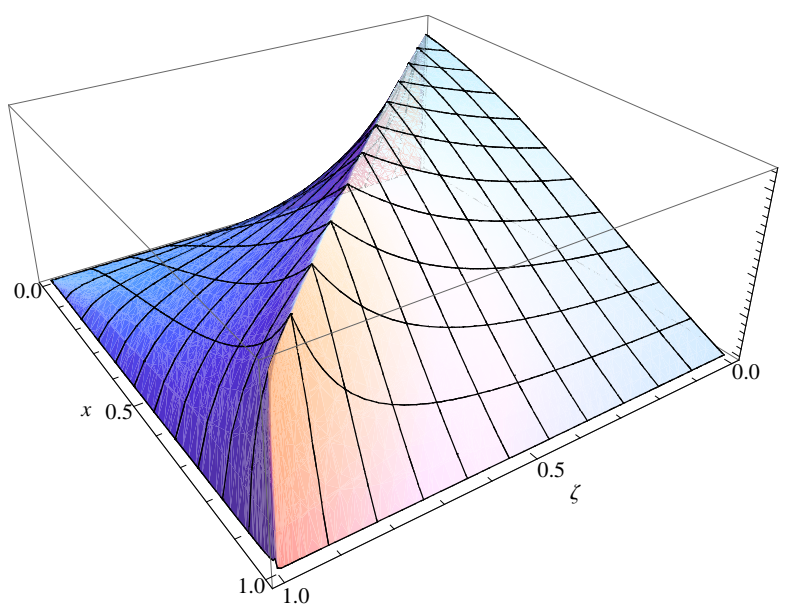

Figure 4: The GPD $\mathscr{H}(x, \zeta)$

kinematical region indeed dominant, but the cat's ears diagram, 'Cat su' is by no means negligible for $Q^{2} \gg M^{2}, m^{2}$. For any value of the momentum transfer larger than the square of the largest mass involved, $m^{2}$, the crossed box 'Box ut' is negligible.

The contribution of the higher Fock states $A_{l l}$, is clearly not negligible for small momentum transfers, but it drops quickly for larger values of $Q^{2}$.

\section{GPD}

A straightforward calculation for the GPD gives

$$
\mathscr{H}=\frac{1}{4 \pi x(1-x)(x-\zeta)\left(M^{2}-\frac{m^{2}}{x(1-x)}\right)\left(\frac{M^{2}}{1-\zeta}-\frac{(1-\zeta) m^{2}}{(1-x)(1-\zeta)}\right)}
$$

in the DGLAP region and

$$
\mathscr{H}=\frac{1}{4 \pi x(1-x)(\zeta-x)\left(M^{2}-\frac{m^{2}}{x(1-x)}\right)\left(\frac{M^{2}}{1-\zeta}-\frac{\zeta m^{2}}{x(\zeta-x)}\right)}
$$

in the ERBL region. (We use the notation $k^{+}=x p^{+}$, where $k^{+}$is the plus-component of the constituent that absorbes the incident virtual photon.) The form factor is given by the sum rule

$$
F(t)=\int_{0}^{1} \mathrm{~d} x \mathscr{H}(x, \zeta)
$$

(Note that in our $1+1$ dimensional model $t$ and $\zeta$ are not independent, see Eq. (2.2). In general, $\mathscr{H}$ is a function of $x, \zeta$, and $t$, as indicated in Eq. (1.1).)

We show the form factor in Fig. 5 together with the result of a realistic model [8]. Although the realistic calculation $(3+1$ dimensions, spinor quarks) differs quantitatively, the qualitative feature that the nonvalence contribution to the form factor dominates for almost all values of $Q^{2}$ remains. 

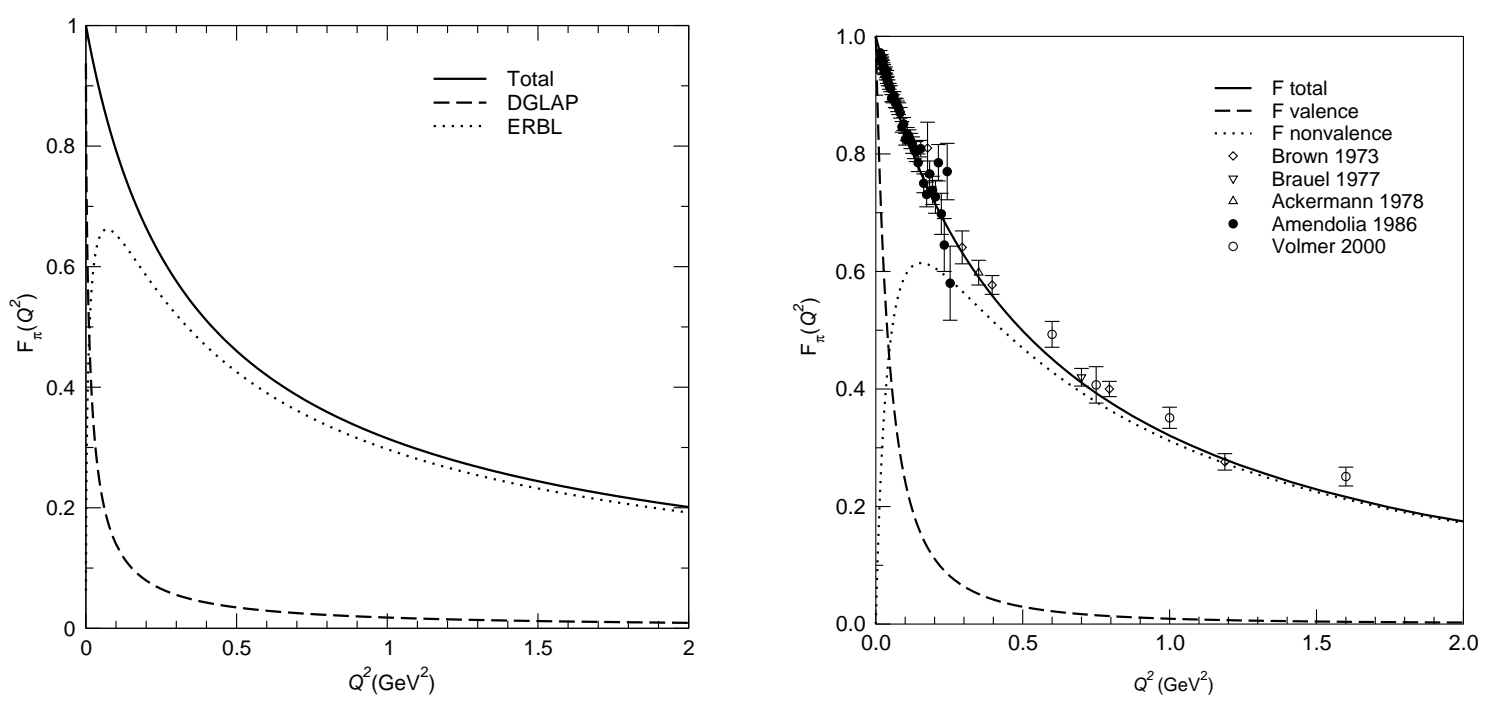

Figure 5: The form factor from the GPD (left) and from a LF calculation in 3+1 dimensions[8] (right).

\section{Spinor Quarks. 1+1 dimensions}

If we take the spin of the constituents into account, the numerator of the integrand in Eq. (2.3) is given by

$$
S^{\mu v}=\operatorname{Tr}\left[\left(\not \psi_{1}+m_{1}\right)\left(\not \psi_{2}+m_{2}\right) \gamma^{\mu}\left(\not \psi_{3}+m_{3}\right) \gamma^{v}\left(\not \psi_{4}+m_{4}\right)\right] .
$$

In $1+1$ dimensions, this reduces to a simple expression

$$
\begin{aligned}
& S^{++}=16 k_{1}^{-} k_{2}^{+} k_{3}^{+} k_{4}^{+}+8\left(m_{1} m_{2} k_{3}^{+} k_{4}^{+}+m_{1} m_{4} k_{2}^{+} k_{3}^{+}+m_{2} m_{4} k_{1}^{+} k_{3}^{+}\right), \\
& S^{+-}=4 m_{1} m_{2} m_{3} m_{4}+8\left(m_{1} m_{3} k_{2}^{+} k_{4}^{-}+m_{2} m_{3} k_{1}^{+} k_{4}^{-}+m_{3} m_{4} k_{1}^{-} k_{2}^{+}\right), \\
& S^{-+}=4 m_{1} m_{2} m_{3} m_{4}+8\left(m_{1} m_{3} k_{2}^{-} k_{4}^{+}+m_{2} m_{3} k_{1}^{-} k_{4}^{+}+m_{3} m_{4} k_{1}^{+} k_{2}^{-}\right), \\
& S^{--}=16 k_{1}^{+} k_{2}^{-} k_{3}^{-} k_{4}^{-}+8\left(m_{1} m_{2} k_{3}^{-} k_{4}^{-}+m_{1} m_{4} k_{2}^{-} k_{3}^{-}+m_{2} m_{4} k_{1}^{-} k_{3}^{-}\right) .
\end{aligned}
$$

If we would calculate these traces in $3+1$ dimensions, additional terms that are of the same or lower order in $k^{-}$would occur. The three $k^{-}$'s cause a LF singularity: An arc contribution is needed. It occurs because the integral over $k^{-}$is divergent, so naively the LF amplitudes cannot be defined. In the context of application of the residue formula, one needs to subtract the contribution from the arc at infinity from the naive residues. It appears that this subtraction produces the correct form of LF amplitudes and the correct support [6]. As the minus components of the momenta are proportional to $k_{\perp}^{2}$, the same terms cause the log divergence of the amplitude in $3+1$ dimensions.

\section{Conclusion and outlook}

\section{Scalar case}

We calculated the GPD of a scalar 'pion' with scalar constituents in a model that we have used before in a calculation of the pion form factor. We showed explicitly that the LF and covariant calculations coincide. 
The relative magnitudes of the LF diagrams corresponding to a covariant amplitude as well as magnitudes of the covariant amplitudes themselves, depend strongly on the value of $Q^{2}$. Because in the kinematic range where we have done our calculations the values of the total momentum squares $s$ are limited to $s<4 m^{2}$, which is not the typical deep-inelastic kinematics, definite conclusions cannot be drawn at this point. We must check our results at $s$-values that are much larger than $m^{2}$ to draw definite conclusions.

\section{Spinor case}

The spin structure gives rise to an arc contribution, that must be taken into account to get full agreement with the covariant amplitude. If we improve our simple model by taking into account the spins of the constituents, we need to regularize in some way in order to render the amplitudes finite. In Ref. [8] we have demonstrated that 'smearing' provides a regularization that is easy to implement and gives realistic results for the pion form factor in a wide range of momentum transfer values.

\section{References}

[1] X. Ji, Phys. Rev. Lett. 78 (1997) 610

[2] A.V. Radyushkin, Phys. Lett. B 380 (1996) 417

[3] J.C. Collins and A. Freund, Phys. Rev. D 59 (1999) 074005

[4] M. Diehl, Phys. Rept. 388 (2003) 41

[5] Chueng-Ryong Ji, Ho-Meoyng Choi, and Bernard L.G. Bakker, Nucl. Phys. A 790 (2007) 583c

[6] B.L.G. Bakker, M.A. DeWitt, C.-R. Ji, and Yu. Mishchenko, Phys. Rev. D 72 (2007) 076005

[7] B.L.G. Bakker and Chueng-Ryong Ji, Phys. Rev. D 62 (2001) 074014

[8] B.L.G. Bakker, Ho-Meoyng Choi, and Chueng-Ryong Ji, Phys. Rev. D 63 (2001) 074014

[9] S. Noguera, L. Theussl, and V. Vento, Eur. Phys. J. A 20 (2004) 483 